\title{
Fidelity susceptibility and conductivity of the current in one-dimensional lattice models with open or periodic boundary conditions
}

\author{
S. Greschner, ${ }^{1}$ A. K. Kolezhuk, ${ }^{2,3}$ and T. Vekua ${ }^{1}$ \\ ${ }^{1}$ Institut für Theoretische Physik, Leibniz Universität Hannover, 30167 Hannover, Germany \\ ${ }^{2}$ Institute of High Technologies, Taras Shevchenko National University of Kiev, 03022 Kiev, Ukraine \\ ${ }^{3}$ Institute of Magnetism, National Academy of Sciences and Ministry of Education, 03142 Kiev, Ukraine \\ (Received 23 August 2013; revised manuscript received 18 October 2013; published 1 November 2013)
}

\begin{abstract}
We study, both numerically and analytically, the finite-size scaling of the fidelity susceptibility $\chi_{J}$ with respect to the charge or spin current in one-dimensional lattice models and relate it to the low-frequency behavior of the corresponding conductivity. It is shown that in gapless systems with open boundary conditions the leading dependence on the system size $L$ stems from the singular part of the conductivity and is quadratic, with a universal form $\chi_{J}=\left[7 \zeta(3) / 2 \pi^{4}\right] K L^{2}$, where $K$ is the Luttinger liquid parameter and $\zeta(x)$ is the Riemann $\zeta$ function. In contrast to that for periodic boundary conditions the leading system size dependence is directly connected to the regular part of the conductivity and is subquadratic, $\chi_{J} \propto L^{\gamma}$, where the $K$-dependent exponent $\gamma$ is equal to 1 in most situations (as a side effect, this relation provides an alternative way to study the low-frequency behavior of the regular part of the conductivity). For open boundary conditions, we also study another current-related quantity, the fidelity susceptibility to the lattice tilt $\chi_{\mathcal{P}}$, and show that it scales as the quartic power of the system size, $\chi_{\mathcal{P}}=\left[31 \zeta(5) / 8 \pi^{6}\right]\left(K L^{4} / u^{2}\right)$, where $u$ is the sound velocity. Thus, the ratio $L^{2} \chi_{J} / \chi_{\mathcal{P}}$ directly measures the sound velocity in open chains. The behavior of the current fidelity susceptibility in gapped phases is discussed, particularly in the topologically ordered Haldane state.
\end{abstract}

DOI: 10.1103/PhysRevB.88.195101

PACS number(s): 71.10.Pm, 75.40.Gb, 64.60.-i, 72.15.Nj

\section{INTRODUCTION}

The ground-state fidelity susceptibility (FS) has established itself as a useful computational tool for locating quantum phase transitions in many-body systems. ${ }^{1-4}$ For a general Hamiltonian,

$$
\widehat{\mathcal{H}}(\lambda)=\widehat{\mathcal{H}}_{0}+\lambda \widehat{W},
$$

with a phase transition driven by the coupling to a certain operator $\widehat{W}$, the fidelity $F(\lambda, \delta \lambda)=\left\langle\psi_{0}(\lambda) \mid \psi_{0}(\lambda+\delta \lambda)\right\rangle$ measures the change in the ground-state wave function $\left|\psi_{0}(\lambda)\right\rangle$ with the infinitesimal change of the coupling $\lambda$, and the fidelity susceptibility $\chi_{W}$ with respect to the "perturbation" $W$ is defined as ${ }^{1,2}$

$$
\begin{aligned}
\chi_{W}(\lambda) & =\lim _{\delta \lambda \rightarrow 0} \frac{1-|F(\lambda, \delta \lambda)|^{2}}{\delta \lambda^{2}} \\
& =\sum_{n \neq 0} \frac{\left|\left\langle\psi_{0}(\lambda)|\widehat{W}| \psi_{n}(\lambda)\right\rangle\right|^{2}}{\left[E_{n}(\lambda)-E_{0}(\lambda)\right]^{2}},
\end{aligned}
$$

where the last line is obtained in the second order of perturbation theory ${ }^{1,2}$ assuming that the ground state is unique. Summation in (2) is over all eigenstates $\left|\psi_{n}(\lambda)\right\rangle$ of the Hamiltonian $\mathcal{H}(\lambda)$ with the eigenvalues $E_{n}(\lambda)$, except the ground state $\left|\psi_{0}(\lambda)\right\rangle$.

Typically, in the thermodynamic limit, i.e., for system size $L \rightarrow \infty$, the FS would become an extensive quantity that diverges at point $\lambda_{c}$, corresponding to a quantum phase transition, $\chi_{W} \propto L /\left(\lambda-\lambda_{c}\right)^{\alpha}$, while for systems of finite size the analysis of the scaling behavior of

$$
\chi_{W}\left(\lambda_{c}\right) \propto L^{\mu}
$$

allows one to extract the critical exponent $v$ of the correlation length, $v=(\mu-1) / \alpha$, and thus to determine the universality class of the transition.
One of the most advanced unbiased numerical methods for analyzing lattice models in reduced spatial dimensions is the density matrix renormalization group ${ }^{6,7}$ (DMRG), which is best suited for systems with open boundary conditions along at least one of the spatial directions. In one dimension (1D), systems with open boundaries consisting of $L \sim 10^{2}-10^{3}$ sites can be efficiently analyzed by DMRG. Hence, it is crucial to understand the dependence of the FS on the boundary conditions. For many types of the perturbation $W$, the FS depends only weakly on the boundary conditions for large systems.

In the present paper, we show that if $\widehat{W}$ is charge- or spincurrent operator $\widehat{J}$ or the "polarization" operator $\widehat{\mathcal{P}}$ (which physically corresponds to introducing the external electric field for charged particles or to tilting the lattice for neutral particles or to a magnetic field gradient for spins), the situation is very special. We study the current FS in several model systems, including spin chains, the Hubbard model for spinful fermions, and the Bose-Hubbard model. It is shown that in gapless 1D systems with open boundary conditions (o.b.c.) the leading terms in the $L$ dependence are given by $\chi_{J} \propto K L^{2}$ and $\chi_{\mathcal{P}} \propto$ $K L^{4} / u^{2}$, where $K$ is the Luttinger liquid parameter, $u$ is the characteristic "sound" velocity, and the numerical prefactors are universal. We show, by means of relating $\chi_{J}$ and $\chi_{\mathcal{P}}$ to the behavior of the positive frequency conductivity $\sigma_{1}(\omega)$, that those superextensive terms in the FS originate from the lowfrequency behavior of the singular part of the conductivity. Since those terms are universal, they can mask the diverging part of the FS at a phase-transition point between two gapless regions.

In contrast, for gapless systems with periodic boundary conditions (p.b.c.) the leading system size dependence of the current FS is linear, $\chi_{J} \propto L$, in a wide range of the Luttinger liquid parameter $K$, and may change to a subquadratic 
one, $\chi_{J} \propto L^{\gamma}$ with $\gamma$ depending on $K, 1 \leqslant \gamma<2$. At the Kosterlitz-Thouless (KT) metal-insulator transition point $\chi_{J} \propto(L / \ln L)^{2}$.

As a by-product of this study, we establish the general properties of the low-frequency behavior of the conductivity in systems with open and periodic boundary conditions. We emphasize a crucial difference in the behavior of the conductivity in systems with p.b.c. and o.b.c., which is responsible for the peculiar difference in the current FS properties.

We also show that in gapped phases the current FS is generically extensive, independent of boundary conditions, $\chi_{J} \propto L$, but it may again acquire the quadratic system size dependence for topologically ordered states in systems with open boundary conditions, for example, in the singlet ground state of the open Haldane chain, due to nonlocally entangled edge spins.

The structure of the paper is as follows: in Sec. II we consider the main properties of the current fidelity susceptibility, its relation to the conductivity, and the dependence on boundary conditions for the simplest example of the spin- $\frac{1}{2}$ $X X Z$ chain in its gapless phase (which is equivalent to nearest-neighbor interacting spinless fermions). We present two ways of calculating the current FS for open systems: one is based on the free-fermion picture and involves bosonization arguments for a generalization to the interacting case, and the other way is based on applying a unitary twist transformation and reducing the problem to calculating certain integrals of the (spin) density correlation function. We also present an example of how the presence of universal quadratic terms in the current FS can hinder the detection of phase transitions between two gapless phases of the 1D Bose-Hubbard model. In Sec. III we consider the properties of the current FS in the fermionic Hubbard model. Section IV discusses the behavior of the current FS in gapped phases, and Sec. V contains a brief summary. In the Appendix we provide details of the bosonization calculations used throughout the paper for open chains.

\section{SPIN-CURRENT FIDELITY SUSCEPTIBILITY AND CONDUCTIVITY OF THE SPIN $-\frac{1}{2} X X Z$ CHAIN}

We start by considering a spin- $\frac{1}{2} X X Z$ chain with the additional Dzyaloshinskii-Moriya (DM) coupling, described by the Hamiltonian

$$
\widehat{H}=\widehat{H}_{\mathrm{XxZ}}+\widehat{H}_{\mathrm{DM}},
$$

with

$$
\begin{aligned}
\widehat{H}_{\mathrm{XXZ}} & =J \sum_{l}\left(S_{l}^{x} S_{l+1}^{x}+S_{l}^{y} S_{l+1}^{y}+\Delta S_{l}^{z} S_{l+1}^{z}\right), \\
\widehat{H}_{D M} & =d \widehat{J}, \quad \widehat{J}=\sum_{l} J_{l}=\sum_{l}\left(\vec{S}_{l} \times \vec{S}_{l+1}\right)^{z},
\end{aligned}
$$

where $S_{l}^{\alpha}$ are spin- $\frac{1}{2}$ operators acting at site $l$ of the chain. In what follows, we set the Planck constant $\hbar$ and the lattice spacing $a$ to unity and measure energy in units of $J=1$.

We will study the current fidelity susceptibility (CFS) $\chi_{J}(d)$ that describes the response of the ground state to an infinitesimal change of the DM coupling $d$. In the following, we study separately the cases of open and periodic boundary conditions. We will use the upper indices $o$ and $p$ to distinguish the FS for those two cases.

Consider first the CFS at $d=0$ (the alternative derivation, valid for finite $d$ and for arbitrary half-integer spin $S$, is presented later in Sec. IID). At $d=0$, the quantity $\widehat{J}$ has the meaning of the total spin current because the local currents $J_{l}$ satisfy the continuity equation $\partial_{t} S_{l}^{z}=J_{l}-J_{l+1}$.

It is worthwhile to note that the CFS is identical to the so-called stiffness $\mathrm{FS}^{8} \chi_{\rho}$ [defined in Eq. (7)], which describes the response of the ground state to a uniform infinitesimal twist $\varphi$ on every link:

$$
\begin{gathered}
\widehat{H} \mapsto \widehat{H}(\varphi)=\sum_{j}\left[\left(\frac{1}{2} S_{j}^{+} S_{j+1}^{-} e^{i \varphi}+\text { H.c. }\right)+\Delta S_{j}^{z} S_{j+1}^{z}\right], \\
\chi_{\rho}=\lim _{\varphi \rightarrow 0} \frac{1-\left|\left\langle\psi_{0}(0) \mid \psi_{0}(\varphi)\right\rangle\right|^{2}}{\varphi^{2}} .
\end{gathered}
$$

The second derivative of the ground-state energy with respect to $\varphi$ defines the spin stiffness $\rho$; exact results for $\rho$ are available for the spin- $\frac{1}{2} X X Z$ chain $^{9}$ as well as for the Hubbard model. ${ }^{9,10}$

Note that, even though $\chi_{J}=\chi_{\rho}$, the second derivatives of the ground-state energy with respect to $d$ and $\varphi$ differ. Following the results of Ref. 9, the second derivative with respect to the twist

$$
\left.\frac{\partial^{2} E_{0}}{\partial \varphi^{2}}\right|_{\varphi=0}=\left\langle-T_{k}\right\rangle-2 \sum_{n \neq 0} \frac{\left|\left\langle\psi_{0}|\widehat{J}| \psi_{n}\right\rangle\right|^{2}}{E_{n}-E_{0}}=2 L \rho
$$

gives the spin stiffness $\rho$, as already mentioned, where $T_{k}=$ $\sum_{j}\left[\frac{1}{2} S_{j}^{+} S_{j+1}^{-}+\right.$H.c. $]$is the kinetic energy. On the other hand, a similar perturbative calculation ${ }^{3}$ for the second derivative with respect to the DM coupling yields

$$
\left.\frac{\partial^{2} E_{0}}{\partial d^{2}}\right|_{d=0}=-2 \sum_{n \neq 0} \frac{\left|\left\langle\psi_{0}|\widehat{J}| \psi_{n}\right\rangle\right|^{2}}{E_{n}-E_{0}} .
$$

In particular, for p.b.c. for $\Delta=0, \partial^{2} E_{0} / \partial d^{2}=0$ as current commutes with kinetic energy in periodic chains and thus current operator and kinetic energy share common eigenstates; however, spin stiffness is nonzero, and $\rho=1 / \pi$ for the free case.

For the systems with o.b.c. the uniform twist can be completely absorbed by unitary transformation for any $\Delta$, and thus

$$
\left.\frac{\partial^{2} E_{0}}{\partial \varphi^{2}}\right|_{\varphi=0}=0
$$

Alternatively, Eq. (10) for open chains follows from the $f$-sum rule [see (18) below]; the same sum rule implies, for the other derivative,

$$
\left.\frac{\partial^{2} E_{0}}{\partial d^{2}}\right|_{d=0}=-2 \sum_{n \neq 0} \frac{\left|\left\langle\psi_{0}|\widehat{J}| \psi_{n}\right\rangle\right|^{2}}{E_{n}-E_{0}}=\left\langle T_{k}\right\rangle,
$$

and it does not vanish even for $\Delta=0\left(\left\langle T_{k}\right\rangle=-L / \pi\right.$ for the free case) as current does not commute with kinetic energy for o.b.c.

It is also worth remarking that if one performs a twist by $L \varphi \ll 1$ on only one link of a periodic chain (twisting the boundary conditions), the energies will be the same as for the 
uniform twist by the angle $\varphi$ on every link (since these two cases are related by canonical transformation), but the FS with respect to the twist on one link will be different ${ }^{8}$ from $\chi_{\rho}$ of Eq. (6). The reason is that twisting the single link (twisting the boundary condition) breaks translational symmetry and thus makes the situation similar to that in the o.b.c. case. As a result, the response of the ground-state wave function to the infinitesimal twist on a single link is nonzero independent of boundary conditions, even in the noninteracting case $(\Delta=0)$.

Instead of the spin- $\frac{1}{2} X X Z$ chain with the DM coupling, described by the Hamiltonian (4), one may have in mind interacting lattice fermions or hard-core bosons under the action of some "field" $d$ that couples to the total particle current,

$$
\begin{aligned}
\widehat{H}= & -\frac{1}{2} \sum_{j}\left[c_{j}^{\dagger} c_{j+1}+c_{j+1}^{\dagger} c_{j}\right]+\Delta \sum_{j} n_{j} n_{j+1} \\
& -i \frac{d}{2} \sum_{j}\left[c_{j}^{\dagger} c_{j+1}-c_{j+1}^{\dagger} c_{j}\right],
\end{aligned}
$$

which is equivalent to the spin- $\frac{1}{2} X X Z$ chain by the wellknown Jordan-Wigner transformation. At $d=0$ CFS $\chi_{J}$ defines the response of the ground state of such a system to the infinitesimal uniform change of current through nearestneighbor links.

\section{A. Relation between the CFS and conductivity}

According to (2), the CFS can be written as

$$
\chi_{J}(d=0)=\sum_{n \neq 0} \frac{\left|\left\langle\psi_{0}|\widehat{J}| \psi_{n}\right\rangle\right|^{2}}{\left(E_{n}-E_{0}\right)^{2}},
$$

where $E_{n}$ are the eigenvalues of $\widehat{H}_{\mathrm{XXZ}}$ and the summation is over all excited states. Comparing the above expression to the definition of the positive-frequency real part of the spin-current conductivity, ${ }^{11}$

$$
\begin{aligned}
\sigma_{1}(\omega) & \left.\equiv \operatorname{Re} \sigma(\omega)\right|_{\omega>0} \\
& =\frac{\pi}{L \omega} \sum_{n \neq 0}\left|\left\langle\psi_{0}|\widehat{J}| \psi_{n}\right\rangle\right|^{2} \delta\left[\omega-\left(E_{n}-E_{0}\right)\right],
\end{aligned}
$$

one obtains the following relation between the CFS and the integrated conductivity:

$$
\chi_{J}=\frac{L}{\pi} \int_{0}^{\infty} d \omega \frac{\sigma_{1}(\omega)}{\omega} .
$$

It is important that for p.b.c. systems definition (14) does not include the Drude weight term $K u \delta(\omega)$. The Drude weight is concentrated at $\omega=0$, while the sum in Eq. (14) is over the energy eigenstates with the lower bound $E_{n}-E_{0} \sim 1 / L>0$, so it does not account for the zero mode. ${ }^{9}$

In contrast, in systems with o.b.c. the total current does not commute with the Hamiltonian even in the noninteracting case $(\Delta=0)$. Its zero mode vanishes identically [see Eq. (A3) in the Appendix]; hence the singular part of the conductivity (the Drude weight term) is included in $\sigma_{1}(\omega)$. As we will see below, it is due to this reason that the finite-size scaling of $\chi_{J}$ is quite different for systems with periodic and open boundary conditions.

\section{B. Periodic boundary conditions}

For a periodic chain

$$
\sigma_{1}^{p}(\omega)=\sigma_{\text {reg }}(\omega),
$$

where $\sigma_{\text {reg }}(\omega)$ is a regular part of the conductivity; as mentioned above, the total real part of the conductivity (including zero mode) is

$$
\operatorname{Re} \sigma^{p}(\omega)=K u \delta(\omega)+\sigma_{\text {reg }}(\omega),
$$

where $u=\frac{K}{2 K-1} \sin \frac{\pi}{2 K}$ has the meaning of the spin-wave velocity of the $X X Z$ chain. The total conductivity satisfies the $f$-sum rule $9,12-14$

$$
\frac{1}{\pi} \int_{0}^{\infty} \sigma(\omega) d \omega=-\frac{1}{2 L}\left\langle T_{k}\right\rangle
$$

where $\left\langle T_{k}\right\rangle$ is the average kinetic energy, which in the case of the $X X Z$ chain can be evaluated as

$$
\left\langle T_{k}\right\rangle=E_{0}(\Delta)-\Delta \partial_{\Delta} E_{0}(\Delta)
$$

from the exact dependence of the ground-state energy on $\Delta .^{15}$

One can observe that for the $X X Z$ chain with $-1<\Delta \lesssim 1 / 4$ the product $K u$ is well approximated by

$$
K u \simeq \pi\left\langle-T_{k}\right\rangle / L,
$$

so the right-hand side of (18) is (in a rather wide region, $-1<\Delta \lesssim 1 / 4)$ well approximated by $K u /(2 \pi)$, and thus, in this region the sum rule (18) is exhausted to a high accuracy by the Drude term [using $\int_{0}^{\infty} \delta(x) d x=1 / 2$ ].

For weak interaction $\Delta$, a perturbative calculation ${ }^{17}$ yields $\sigma_{\text {reg }}(\omega) \sim \Delta^{2} \omega^{8 K-5}$. Then for $K>5 / 8$ (which corresponds to $\Delta<\frac{1+\sqrt{5}}{4} \simeq 0.8$ and, strictly speaking, is outside the perturbative regime in $\Delta$ ) the integral in (15) is $O(1)$, so the CFS has a usual extensive dependence on the system size,

$$
\chi_{J}^{p} \propto L+\cdots, \quad K>\frac{5}{8},
$$

where the dots (here and throughout the paper) stand for subleading contributions in the system size. The situation is different for periodic chains with $1 / 2 \leqslant K \leqslant 5 / 8$, where relation (15) suggests the following nontrivial dependence on the system size:

$$
\chi_{J}^{p} \propto L(\ln L+\text { const }) \quad \text { at } \quad K=5 / 8
$$

and

$$
\chi_{J}^{p} \propto L^{6-8 K}+\cdots, \quad \frac{1}{2}<K<\frac{5}{8} .
$$

The KT phase-transition point $\Delta=1$, where $K=1 / 2$, must be treated separately since at this point the conductivity gets logarithmic corrections, ${ }^{11} \sigma_{\text {reg }}(\omega) \sim 1 / \omega \ln ^{2}(\omega)$, and hence

$$
\begin{aligned}
\chi_{J}^{p} & \propto L\left[\frac{\operatorname{li}(A L)}{A}-\frac{L}{\ln (A L)}+\text { const }\right]+\cdots \\
& \sim(L / \ln L)^{2}+\cdots \text { at } \Delta=1,
\end{aligned}
$$

with some numerical coefficient $A$ and $\operatorname{li}(x)$ being the logarithmic integral function. Equations (22)-(24) can be obtained by replacing the lower integration limit in Eq. (15) by a quantity of the order of $u / L$. 


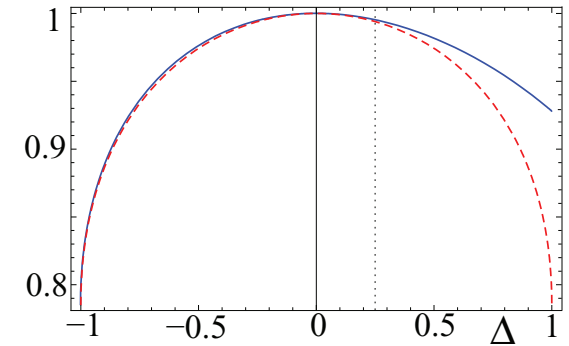

FIG. 1. (Color online) Exact results for $-\frac{\pi}{L}\left\langle T_{k}\right\rangle$ (solid line) vs $K u$ (dashed line) in the spin- $\frac{1}{2} X X Z$ chain as a function of the anisotropy $\Delta$ obtained from the Bethe ansatz solution in the thermodynamic limit. The deviation at $1 / 4 \lesssim \Delta$ shows that in this region the regular part of the conductivity has to contribute noticeably to the sum rule (18). The Drude weight (the coefficient in front of the $\delta$ function describing the ballistic transport) is related to the spin stiffness as $K u=\pi \rho .^{9,10,16}$

It should be remarked that our results [Eqs. (21)-(24)] disagree with the analytical estimates presented in Ref. 8 for the stiffness FS $\chi_{\rho}$ that is equal to our current FS $\chi_{J}$, as already mentioned. Namely, Eq. (20) of Ref. 8 creates the impression that the leading contribution to $\chi_{\rho}$ scales generically as $L^{2}$ in periodic systems. This, in light of the intimate connection between the CFS and the regular part of the conductivity established by us above, would require $\sigma_{\text {reg }}(\omega) \sim 1 / \omega$ at low frequencies, contradicting the $f$-sum rule for the conductivity. In addition the $K$-dependent contribution presented in Eq. (20) of Ref. 8 also disagrees with our $K$-dependent contribution given in Eqs. (22)-(24).

Figure 2 shows the DMRG results for $\chi_{J}^{p}$ in periodic $X X Z$ chains with different values of the interaction $\Delta$. For $\Delta \leqslant 0.5$, a good convergence to the linear scaling $\chi_{J}^{p} / L \propto$ const $+O(1 / L)$ is achieved. However, for $0.8>\Delta>0.5$ we cannot confirm reliably convergence of $\chi_{J}^{p} / L$ to a constant for the system sizes studied, $L<100$. At $\Delta=0.8(K=5 / 8)$ we fitted well our numerical data according to Eq. (22). In the region $0.8<\Delta<1$ our fits reproduce nicely the leading

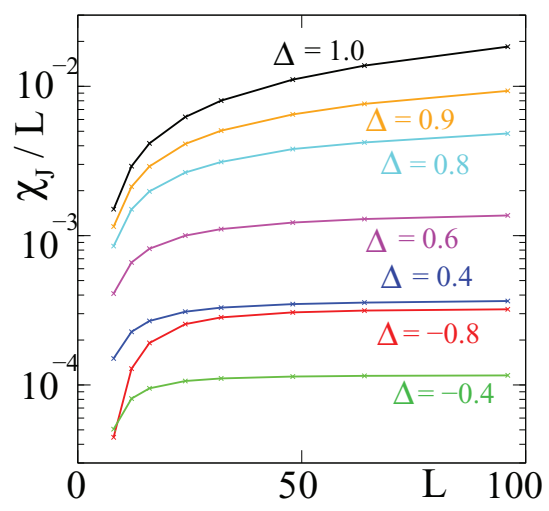

FIG. 2. (Color online) The finite-size scaling of the current FS $\chi_{J}$ in the spin- $\frac{1}{2} X X Z$ chain with p.b.c. for different values of the anisotropy $\Delta$. In DMRG simulations, we have chosen the step $\delta d=$ $10^{-3}$ and have kept about $m \sim 1000$ states to achieve good accuracy. For small system sizes $L \leqslant 26$ our DMRG data reproduced Lanczos results presented in Figs. 1 and 2 of Ref. 8 (note that there is a misprint on the vertical axis labeling of Fig. 2 in Ref. 8).

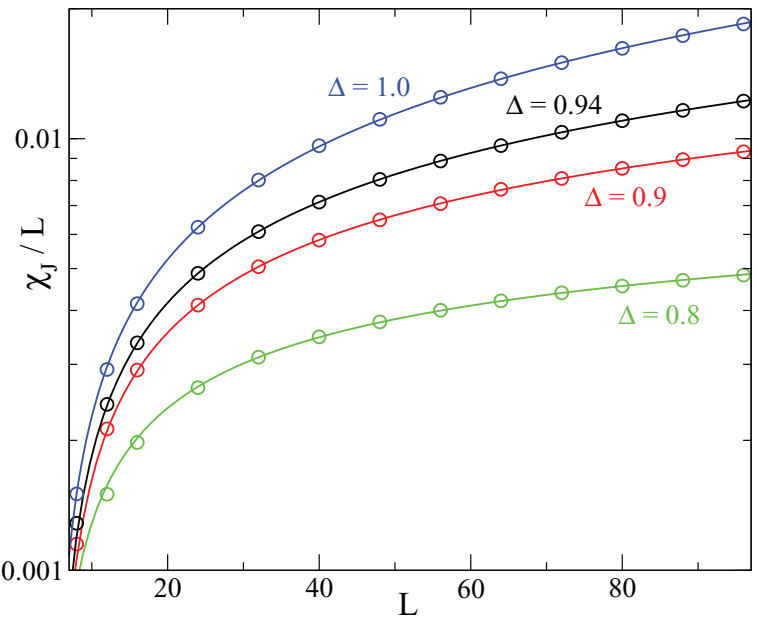

FIG. 3. (Color online) Fits of the numerical data shown in Fig. 2 (circles) for $\Delta \geqslant 0.8$ according to Eqs. (22)-(24). At $\Delta=0.8$ the data are fitted to Eq. (22), $10^{3} \chi_{J}^{p} / L=1.57 \ln L-2.33$. At $\Delta=0.9$ and $\Delta=0.94$ the data are fitted to Eq. (23) with subleading corrections: $\Delta=0.9,10^{3} \chi_{J}^{p} / L=2.46 L^{0.33}+0.84 \ln L-5.55$ and $\Delta=0.94$, $10^{3} \chi_{J}^{p} / L=1.17 \sqrt{L}+1.11 \ln L-4.44$. Finally, at $\Delta=1$ the data are fitted to Eq. (24), $10^{3} \chi_{J}^{p} / L=4.91[\operatorname{li}(4 L) / 4-L / \ln (4 L)]-$ 3.86. Numerical error is estimated to be smaller than the circle size.

nontrivial system size dependence in Eq. (23) when including the subleading corrections of the form of Eq. (22). Finally, at $\Delta=1$ a good fit of $\chi_{J}^{p}$ by Eq. (24) with $A \simeq 4$ is observed. We present our fits for $0.8 \leqslant \Delta \leqslant 1$ in Fig. 3 .

At $\Delta=1$ it is worthwhile to mention the effect of the next-nearest-neighbor antiferromagnetic coupling $J_{2}$ (which is also assumed to be $S U(2)$ symmetric),

$$
\widehat{H}=\sum_{j}\left(\vec{S}_{j} \vec{S}_{j+1}+J_{2} \vec{S}_{j} \vec{S}_{j+2}\right)
$$

One can observe that due to $J_{2}$ coupling, the expression of current operator changes as follows:

$$
\widehat{J} \rightarrow \frac{i}{2} \sum_{j}\left(S_{j}^{+} S_{j+1}^{-}+J_{2} S_{j}^{+} S_{j+2}^{-}\right)+\text {H.c. }
$$

At the special point $J_{2}=J_{2}^{c} \simeq 0.241$ (that in the thermodynamic limit corresponds to the phase transition between the Luttinger liquid and dimerized phases) the amplitude of the basic (marginal) umklapp term vanishes in the effective bosonization formulation. For that reason, the low-frequency behavior of the regular part of the conductivity changes to $\sigma_{1}(\omega) \sim \omega^{8 n K-5}$, where $K=1 / 2$ due to $S U(2)$ symmetry and $n>1$ is some integer, so that in any case the integral $\int_{0}^{\infty}\left[\sigma_{1}(\omega) / \omega\right] d \omega$ converges at $\omega=0$. Hence at $J_{2}=J_{2}^{c}$ the CFS $\chi_{J}^{p} \sim L$, even though this is a phase-transition point between a gapless and a gapped region. This agrees well with the data shown in Fig. 4 of Ref. 8; namely, the ratio $\chi_{J}^{p} / L$ becomes nearly system size independent at $J_{2}=J_{2}^{c}$. The form of the current operator (26) explains why the infinitesimal twist in Ref. 8 has to be the same in both nearest-neighbor and next-nearest-neighbor links to observe the flat curve $\chi_{J}^{p} / L$ vs $L$ at $J_{2}=J_{2}^{c}$. 


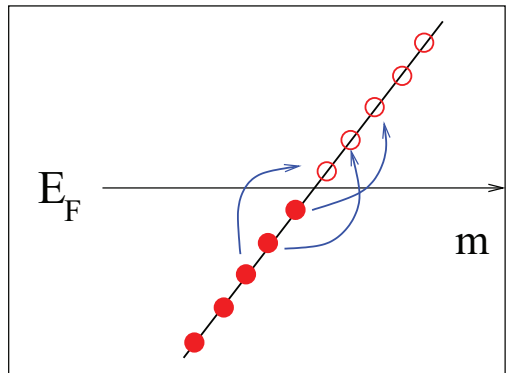

FIG. 4. (Color online) An example of the particle-hole excitation with excitation energy $3 \pi u / L$. As one can see, there are exactly $\rho(3)=3$ different particle-hole excitations (involving the creation of a single particle-hole pair) with that energy. A similar picture holds for any energy $E_{m}-E_{0}=\pi u m / L$; hence, $\rho(m)=m$.

\section{Open boundary conditions}

Let us start our discussion of the CFS for open chains from the noninteracting case $\Delta=0$ (free spinless fermions or hardcore bosons). At low excitation energies, the spectrum is approximately linear, $E_{m}-E_{0} \simeq u \pi m / L$. Expression (14), which for o.b.c. represents the entire conductivity, can be rewritten as

$$
\sigma_{1}^{o}=\frac{\pi}{\omega L} \sum_{m>0} \rho(m)\left|\left\langle\psi_{0}|\widehat{J}| \psi_{m}\right\rangle\right|^{2} \delta\left[\omega-\left(E_{m}-E_{0}\right)\right],
$$

where the matrix element of the current (see the Appendix) is

$$
\left|\left\langle\psi_{0}|\widehat{J}| \psi_{m}\right\rangle\right|=\frac{\left[1-(-1)^{m}\right] u}{m \pi},
$$

where, according to our conventions for free fermions, $u(\Delta=0)=1$ and the degeneracy

$$
\rho(m)=m
$$

is the number of different particle-hole excitations with the same energy $E_{m}$ as is illustrated in Fig. 4 (excited states with more than one particle-hole pair do not contribute since they cannot be created by the current operator from the ground state). Note that the matrix element satisfies the parity selection rule and is nonzero only for odd $m=2 k+1$.

Putting everything together, we obtain the following lowfrequency behavior for the conductivity of free fermions (the $X Y$ model, $\Delta=0$ ) in an open chain:

$$
\begin{aligned}
\sigma_{1}^{o} & =\frac{\pi u^{2}}{L \omega} \sum_{k=0}^{\infty} \frac{4(2 k+1)}{(2 k+1)^{2} \pi^{2}} \delta\left[\omega-\left(E_{2 k+1}-E_{0}\right)\right] \\
& =\sum_{k=0}^{\infty} \frac{4 u}{(2 k+1)^{2} \pi^{2}} \delta\left(\omega-\frac{(2 k+1) u \pi}{L}\right) .
\end{aligned}
$$

Note that this low-frequency behavior is singular, $L \sigma_{1}^{o}(\omega) \propto$ $1 / \omega^{2}$ at $\omega \rightarrow 0$. This is the form into which the Drude peak transforms in the open chain.

For the interacting case, we divide the conductivity of the open chain into the Drude part $D(\omega)$ and the "regular" contribution,

$$
\sigma_{1}^{o}(\Delta)=D(\omega)+\sigma_{\text {reg }}^{o} .
$$

We calculate the Drude part within the Luttinger liquid (LL) approximation (i.e., we neglect umklapp processes). For the in- teracting case, the expression for the current operator does not change since the interaction commutes with the local-density operator, but the matrix elements of the current equation (28) do change. To separate the Drude contribution, we estimate the matrix element of the current for the interacting case. Introducing a bosonic field $\Phi$ and its conjugate momentum $\Pi$, which satisfy the commutation relations $[\Phi(x), \Pi(y)]=$ $i \delta(x-y)$, we get the following Gaussian model as the effective bosonic Hamiltonian of free fermions:

$$
\widehat{H}(\Delta=0)=\frac{u(\Delta=0)}{2} \int_{0}^{L} d x\left[\left(\partial_{x} \Phi\right)^{2}+\Pi^{2}\right] .
$$

In the LL approach, the presence of the interaction $\Delta$ leads simply to the rescaling of the bosonic field $\Phi \rightarrow \tilde{\Phi}=\sqrt{K} \Phi$ and modification of the sound velocity having the following rescaling effect on the current:

$\widehat{J} \rightarrow \int_{0}^{L} \frac{\partial_{t} \Phi}{\sqrt{\pi}} d x=\sqrt{K} \int_{0}^{L} \frac{\partial_{t} \tilde{\Phi}}{\sqrt{\pi}} d x=\sqrt{K} u \int_{0}^{L} \frac{\tilde{\Pi}}{\sqrt{\pi}} d x$, and the effective LL Hamiltonian of interacting fermions is

$$
\begin{aligned}
\widehat{H}_{X X Z} & =\widehat{H}(\Delta=0)+\Delta \sum_{i} S_{l}^{z} S_{l+1}^{z} \\
& \mapsto \widehat{H}_{L L}=\frac{u(\Delta)}{2} \int_{0}^{L}\left[\left(\partial_{x} \tilde{\Phi}\right)^{2}+\tilde{\Pi}^{2}\right] d x .
\end{aligned}
$$

The matrix element of $\int_{0}^{L} \tilde{\Pi} d x$ between the ground state and excited states is calculated in the Appendix.

Thus, the effect of interactions on the Drude weight (the singular contribution) boils down to rescaling of the matrix elements of the total current for the free case (28) by the factor $\sqrt{K}$, and of course the sound velocity $u$ is also renormalized by the interaction

$$
D(\omega)=\frac{\pi}{L \omega} \sum_{k=0}^{\infty} \frac{4 K u^{2}}{(2 k+1) \pi^{2}} \delta\left(\omega-\frac{(2 k+1) u \pi}{L}\right) .
$$

The integral of the Drude part $D(\omega)$ is exactly equal to that of the Drude weight in a periodic chain:

$$
\int_{0}^{\infty} D(\omega) d \omega=\sum_{K} \frac{4 K u}{(2 k+1)^{2} \pi^{2}}=\frac{K u}{2} .
$$

Needless to say, as in periodic chains, the singular part $D(\omega)$ almost exhausts the sum rule (18) for $\Delta \lesssim 1 / 4$. The regular part of the conductivity in open and periodic chains can, generally speaking, be different, but the sum rule requires that

$$
\lim _{L \rightarrow \infty} \int_{0}^{\infty}\left[\sigma_{\text {reg }}^{o}-\sigma_{\text {reg }}\right] d \omega \rightarrow 0 .
$$

Importantly, the leading size dependence of the CFS comes from the singular part $D(\omega)$, and thus, in an open chain the CFS scales quadratically with the system size:

$$
\chi_{J}^{o}=\frac{L}{\pi} \int_{0}^{\infty} d \omega \frac{D(\omega)}{\omega}=\sum_{k=0} \frac{4 K L^{2}}{(2 k+1)^{3} \pi^{4}}=\frac{7 \zeta(3)}{2 \pi^{4}} K L^{2} .
$$

Subleading corrections to the leading $L^{2}$ behavior follow both from the regular part $\sigma_{\text {reg }}^{o}$ and from the singular part 
$D(\omega)$ due to the nonlinearity of the spectrum. This result prominently illustrates the difference between the periodic and open chains and shows that one has to be careful when applying the CFS for detecting phase transitions: unless the transition involves some divergences in the current correlators, the leading contribution (35) will be "blind" to it, so the divergence of $\chi_{J}$ at the phase transition will be hidden in the subleading terms [usually, the exponent $\mu$ that determines the finite-size scaling of the divergent part of the FS at the phase transition is some number between 1 and 2; see Eq. (3)].

We illustrate such "masking" on the example of the attractive single-component Bose-Hubbard model with the additional three-body occupation constraint: ${ }^{18}$

$$
\begin{aligned}
H= & -\frac{t}{2} \sum_{j}\left[b_{j}^{\dagger} b_{j+1}+b_{j+1}^{\dagger} b_{j}\right]-\frac{i d}{2} \sum_{j}\left[b_{j}^{\dagger} b_{j+1}-b_{j+1}^{\dagger} b_{j}\right] \\
& +\frac{U}{2} \sum_{j} n_{j}\left(n_{j}-1\right)+U_{3} \sum_{j} n_{j}\left(n_{j}-1\right)\left(n_{j}-2\right),
\end{aligned}
$$

where $b_{j}^{\dagger}$ and $b_{j}$ are the bosonic creation and annihilation operators of particles at site $j, n_{j}=b_{j}^{\dagger} b_{j}$, and the threebody coupling constant $U_{3} \rightarrow \infty$ forbids sites with more than double occupancy. Figure 5 presents the DMRG results for the FS study of the Ising phase transition between the single-particle superfluid and pair superfluid states (see the phase diagram in Ref. 18). The transition is easily detected by looking at the FS with respect to the hopping part (changing $t$ ), but when it is studied by looking at the current FS (i.e., the parameter $d$ is changed), it is masked for chains with o.b.c., as seen in the bottom panel of Fig. 5.

\section{Alternative derivation of the CFS scaling for open boundary conditions}

The CFS behavior in a spin- $S X X Z$ chain with arbitrary half integer $S$ can be analyzed with the help of a different approach, valid at any $d$ as well as at finite magnetization $M$ (i.e., in the presence of some external magnetic field). Consider a unitary transformation defined by the twist operator

$$
\widehat{U}[\phi(d)]=e^{i \phi(d) \widehat{\mathcal{P}}}, \quad \widehat{\mathcal{P}}=\sum_{j} j S_{j}^{z},
$$

where $\phi(d)=\arctan (d)$ and $\widehat{\mathcal{P}}$ is the polarization operator, or the "spin center of mass." Applied to the Hamiltonian (4), it removes the DM interaction for the price of changing the anisotropy. Performing two such transformations, $U(d)$ and $U(d+\delta d)$, one can transform the fidelity $F(d, \delta d)$ into a matrix element of the form

$$
F(d, \delta d)=\left\langle\psi_{0}\left(M, \widetilde{\Delta}_{d}\right)\left|e^{i \delta \phi(d) \widehat{\mathcal{P}}}\right| \psi_{0}\left(M, \widetilde{\Delta}_{d+\delta d}\right)\right\rangle,
$$

where $\delta \phi(d)=\phi(d+\delta d)-\phi(d)$ and $\widetilde{\Delta}_{d}=\Delta / \sqrt{1+d^{2}}$. Expanding the fidelity up to quadratic terms in $\delta d$, one obtains

$$
\begin{aligned}
F(d, \delta d) \simeq & 1-\frac{1}{2} \delta \Delta^{2} \chi_{\Delta}+i \delta \phi\left\langle\psi_{0}\left(M, \widetilde{\Delta}_{d}\right)|\widehat{\mathcal{P}}| \psi_{0}\left(M, \widetilde{\Delta}_{d+\delta d}\right)\right\rangle \\
& -\frac{1}{2} \delta \phi^{2}\left\langle\psi_{0}\left(M, \widetilde{\Delta}_{d}\right)\left|\widehat{\mathcal{P}}^{2}\right| \psi_{0}\left(M, \widetilde{\Delta}_{d+\delta d}\right)\right\rangle
\end{aligned}
$$
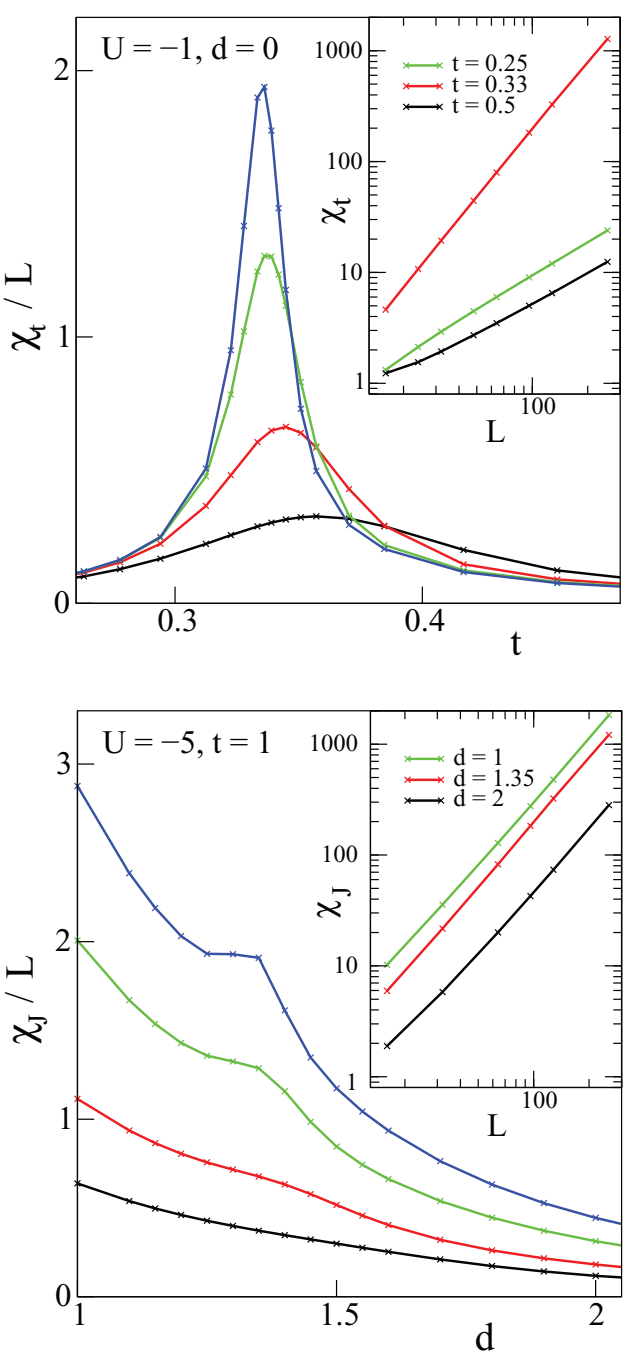

FIG. 5. (Color online) Two different fidelity susceptibilities in the Bose-Hubbard model with o.b.c. (36): (top) the FS $\chi_{t}$ with respect to the hopping $t$ and (bottom) the current FS $\chi_{J}$ for open chains with $L=16,32,64$, and 96 sites (the curves from bottom to top). While $\chi_{t}$ reveals nicely the underlying Ising phase transition between pair and single-particle condensates, in $\chi_{J}$ the finite-size scaling of the peak is masked by its wings: both of them scale as $\sim L^{2}$.

where $\delta \phi=\delta d /\left(1+d^{2}\right), \delta \Delta=-\frac{1}{2} \Delta\left(1+d^{2}\right)^{-3 / 2} \delta d$, and $\chi_{\Delta}$ is the FS with respect to the anisotropy,

$$
\chi_{\Delta}=\lim _{\delta \Delta \rightarrow 0} \frac{1-|F(\Delta, \delta \Delta)|^{2}}{\delta \Delta^{2}} .
$$

$\chi_{\Delta}$ scales with the system size $L$ in a standard way, ${ }^{19}$ i.e., linearly, so it can be neglected if we are interested only in the leading $L$ dependence of $\chi_{J}$, which, as we can already guess, is quadratic.

We will further assume that the total $z$ projection of the spin $S_{\text {tot }}^{z}=\sum_{j} S_{j}^{z}=M L$ is a good quantum number; then even at finite magnetization the ground-state wave function $\left|\psi_{0}(M, \widetilde{\Delta})\right\rangle$ can be made real, so that the geometric connection term

$$
\left\langle\psi_{0}\left(M, \widetilde{\Delta}_{d}\right)|\widehat{\mathcal{P}}| \psi_{0}\left(M, \widetilde{\Delta}_{d+\delta d}\right)\right\rangle-(\text { H.c. })
$$


vanishes. Then the leading term in the current FS can be written as

$$
\chi_{J}^{o}(d, M) \simeq \frac{1}{\left(1+d^{2}\right)^{2}}\left(\left\langle\widehat{\mathcal{P}}^{2}\right\rangle-\langle\widehat{\mathcal{P}}\rangle^{2}\right),
$$

where the averages here and in what follows are taken in the ground state $\left|\psi_{0}\left(M, \widetilde{\Delta}_{d}\right)\right\rangle$. This in turn leads to the formula

$$
\chi_{J}^{o}(d, M)=\frac{\sum_{j, j^{\prime}=1}^{L}\left(j-j^{\prime}\right)^{2}\left(\left\langle S_{j}^{z}\right\rangle\left\langle S_{j^{\prime}}^{z}\right\rangle-\left\langle S_{j}^{z} S_{j^{\prime}}^{z}\right\rangle\right)}{2\left(1+d^{2}\right)^{2}},
$$

where we have again used the assumption that $S_{\text {tot }}^{z}$ is conserved, and hence,

$$
\sum_{j, j^{\prime}} j^{2}\left(\left\langle S_{j}^{z} S_{j^{\prime}}^{z}\right\rangle-\left\langle S_{j}^{z}\right\rangle\left\langle S_{j^{\prime}}^{z}\right\rangle\right)=0 .
$$

Note that the evaluation of the CFS is simplified drastically for open boundary conditions since it is reduced to the task of calculating the spin-spin correlation functions in the ground state.

Only the smooth part of the correlation function $\left\langle S_{j}^{z} S_{j^{\prime}}^{z}\right\rangle$ contributes to the leading size dependence of $\chi_{J}^{o}$. This smooth part has the following universal behavior ${ }^{20}$ (see also Ref. 21, where exact correlation functions for an open spin- $\frac{1}{2} X Y$ chain have been calculated):

$$
\begin{aligned}
\left\langle S_{j}^{z} S_{j^{\prime}}^{z}\right\rangle-\left\langle S_{j}^{z}\right\rangle\left\langle S_{j^{\prime}}^{z}\right\rangle & =-\frac{K}{2 \pi^{2}}\left[\frac{1}{f_{2}\left(j-j^{\prime}\right)}+\frac{1}{f_{2}\left(j+j^{\prime}\right)}\right]+\cdots, \\
f_{2}(x) & =\left[\frac{2(L+1)}{\pi} \sin \frac{\pi x}{2(L+1)}\right]^{2}
\end{aligned}
$$

where $(\cdots)$ denotes oscillating terms and $K=K(M, \widetilde{\Delta})$ is the Luttinger liquid parameter that depends on the effective anisotropy $\widetilde{\Delta}$ and the magnetization per site $M$. For $S=\frac{1}{2}$ and $M=0$, it is given by

$$
K(M=0, \widetilde{\Delta})=\frac{\pi}{2 \arccos (-\widetilde{\Delta})}, \quad \widetilde{\Delta}=\frac{\Delta}{\sqrt{1+d^{2}}} .
$$

In the limit $L \rightarrow \infty$ one can transform the sums in (41) into integrals. Introducing the relative and center-of-mass coordinates, $r=j-j^{\prime}$ and $R=\left(j+j^{\prime}\right) / 2$, we get

$$
\begin{aligned}
\sum_{j, j^{\prime}} \frac{\left(j-j^{\prime}\right)^{2}}{f_{2}\left(j-j^{\prime}\right)} & \rightarrow 2 \int_{0}^{L-r} d R \int_{0}^{L} d r \frac{r^{2}}{f_{2}(r)} \\
& =2 \int_{0}^{L} d r \frac{(L-r) r^{2}}{f_{2}(r)} \\
& =\frac{4 L^{2}}{\pi} \int_{0}^{\pi / 2} d y \frac{(1-2 y / \pi) y^{2}}{\sin ^{2} y} \\
& =\frac{4 L^{2}}{\pi^{2}}\left[-\frac{1}{2} \pi^{2} \ln 2+\frac{21}{4} \zeta(3)\right]
\end{aligned}
$$

and

$$
\begin{aligned}
\sum_{j, j^{\prime}} \frac{\left(j-j^{\prime}\right)^{2}}{f_{2}\left(j+j^{\prime}\right)} & \rightarrow 2 \int_{0}^{L / 2} d R \int_{-2 R}^{2 R} d r \frac{r^{2}}{f_{2}(2 R)} \\
& =\frac{4}{3} \int_{0}^{L / 2} d R \frac{(2 R)^{3}}{f_{2}(2 R)}=\frac{8 L^{2}}{3 \pi^{2}} \int_{0}^{\pi / 2} d y \frac{y^{3}}{\sin ^{2} y} \\
& =\frac{L^{2}}{\pi^{2}}\left[2 \pi^{2} \ln 2-7 \zeta(3)\right] .
\end{aligned}
$$

We obtain the final result for the system size dependence of the current FS in the gapless spin- $\frac{1}{2} X X Z$ chain with open boundaries in the following form:

$$
\frac{\chi_{J}^{o}(d, M)}{L^{2}}=\frac{7 \zeta(3)}{2 \pi^{4}\left(1+d^{2}\right)^{2}} K(M, \widetilde{\Delta})+\cdots .
$$

For an $S=\frac{1}{2}$ chain at zero magnetization, one can use formula (43) for the LL parameter to obtain a closed expression for the CFS; it is easy to see that $\chi_{J}^{o}(d=0, M=0)$ has a singular behavior at $\Delta=1$.

The $L^{2}$ dependence of the current FS is a generic feature for gapless models with o.b.c. and conserved $S^{z}$, where one can eliminate the current term (the DM interaction) by means of a unitary "twist" operator (37) and where the smooth part of the $\left\langle S_{j}^{z} S_{j^{\prime}}^{z}\right\rangle$ correlator decays like $1 /\left|j-j^{\prime}\right|^{2}$.

\section{E. Relation to the tilt fidelity susceptibility}

In a spin chain with open boundaries, one can study another quantity, which is, as we will show, related to the current FS, namely, the fidelity susceptibility $\chi_{\mathcal{P}}$ with respect to the polarization operator $\widehat{\mathcal{P}}=\sum_{j} j S_{j}^{z}$. For a spin- $\frac{1}{2}$ chain, this physically means a response to the gradient of the external magnetic field. For the equivalent system of spinless fermions this could be a response to the the "lattice tilt," or if one assumes that the particles are electrically charged, then this is a response to the external electric field. The tilt FS is given by

$$
\chi_{\mathcal{P}}=\sum_{n \neq 0} \frac{\left|\left\langle\psi_{0}|\widehat{\mathcal{P}}| \psi_{n}\right\rangle\right|^{2}}{\left(E_{n}-E_{0}\right)^{2}} \text {. }
$$

It is easy to see that the tilt FS is related to the "dynamic polarizability" $\alpha(\omega)$,

$$
\alpha(\omega)=\frac{\pi}{L} \sum_{n \neq 0}\left|\left\langle\psi_{0}|\widehat{\mathcal{P}}| \psi_{n}\right\rangle\right|^{2} \delta\left[\omega-\left(E_{n}-E_{0}\right)\right],
$$

by the following formula:

$$
\chi_{\mathcal{P}}=\frac{L}{\pi} \int_{0}^{\infty} d \omega \frac{\alpha(\omega)}{\omega^{2}} .
$$

On the other hand, one has

$$
i\left[\widehat{H}_{\mathrm{XXZ}}, \widehat{\mathcal{P}}\right]=\sum_{l} l\left(J_{l}-J_{l+1}\right)=\widehat{J},
$$

and thus $\sigma_{1}(\omega)=\omega \alpha(\omega)$, which leads to the following relation between the tilt FS and the conductivity:

$$
\chi_{\mathcal{P}}=\frac{L}{\pi} \int_{0}^{\infty} d \omega \frac{\sigma_{1}(\omega)}{\omega^{3}} .
$$

Thus, the leading term in the finite-size scaling of $\chi_{\mathcal{P}}$, similar to the CFS $\chi_{J}$, will be determined just by the low-frequency behavior of the conductivity. Using the formulas for the conductivity, (31) and (33), one arrives at the following result:

$$
\chi_{\mathcal{P}}=\frac{31 K L^{4} \zeta(5)}{8 u^{2} \pi^{6}} .
$$

For free fermions $(\Delta=0)$, the above result can also be reproduced directly in the same way as done in Eqs. (27)-(30) for the conductivity, using the "density of states" (29) and the 
explicit expression for the matrix element (see the Appendix),

$$
\left|\left\langle\psi_{0}|\widehat{\mathcal{P}}| \psi_{m}\right\rangle\right|=\frac{L\left[1-(-1)^{m}\right]}{(m \pi)^{2}} .
$$

Indeed, using the perturbative expression

$$
\chi_{\mathcal{P}}=\sum_{m>0} \rho(m) \frac{\left|\left\langle\psi_{0}|\widehat{\mathcal{P}}| \psi_{m}\right\rangle\right|^{2}}{\left(E_{m}-E_{0}\right)^{2}}
$$

with the linearized spectrum $E_{m}-E_{0} \simeq u \pi m / L$, one obtains for free fermions

$$
\chi_{\mathcal{P}}(\Delta=0)=\sum_{k=0}^{\infty} \frac{4(2 k+1) L^{2}}{\left(E_{2 k+1}-E_{0}\right)^{2}(2 k+1)^{4} \pi^{4}}=\frac{31 L^{4} \zeta(5)}{8 u^{2} \pi^{6}} .
$$

For interacting fermions $S^{z}$ gets the additional factor of $\sqrt{K}$ (see the Appendix), hence bringing us back to the general result (51).

For the ratio of the current FS and the tilt FS in open chains one obtains the universal result

$$
\frac{L^{2} \chi_{J}}{\chi_{\mathcal{P}}}=\frac{28 u^{2} \pi^{2} \zeta(3)}{31 \zeta(5)} \simeq 10.3341 u^{2}
$$

\section{CURRENT FS IN THE FERMIONIC HUBBARD MODEL}

Consider the Hubbard model for spin- $\frac{1}{2}$ fermions (attractive or repulsive) at arbitrary filling:

$$
\widehat{H}_{0}=-\sum_{j, \sigma}\left(c_{j, \sigma}^{\dagger} c_{j+1, \sigma}+\text { H.c. }\right)+U \sum_{j} n_{i}^{2},
$$

where $c_{j, \sigma}$ annihilates a fermion at site $j$ with the $\operatorname{spin} \sigma=$ $\{\uparrow, \downarrow\}$ and $n_{j}=\sum_{\sigma} c_{j, \sigma}^{\dagger} c_{j, \sigma}$ is the fermion density at the site. We assume open boundary conditions and study the FS $\chi_{J}^{o}$ with respect to the total current, $\widehat{H}=\widehat{H}_{0}+\lambda \widehat{J}_{\text {tot }}$, with

$$
\widehat{J}_{\mathrm{tot}}=-i \sum_{j, \sigma}\left(c_{j, \sigma}^{\dagger} c_{j+1, \sigma}-c_{j+1, \sigma}^{\dagger} c_{j, \sigma}\right) .
$$

The CFS can be calculated using the method of the unitary twist operator, as described in Sect. IID for spin chains, with the replacement of $S_{j}^{z}$ by $n_{j}$. One can closely follow all the steps of the calculation presented above for a spin chain and express the CFS through the density-density correlation function of the Hubbard model. Assuming that its smooth part has a form similar to Eq. (42), with $K$ now being the charge Luttinger parameter $K_{c}$ of the Hubbard model, we obtain the leading term in the finite-size scaling of the CFS as follows:

$$
\frac{\chi_{J}^{o}}{L^{2}}=\frac{7 \zeta(3)}{\pi^{4}} K_{c}(v, M)+\cdots,
$$

where $v$ is the lattice filling and $M$ is the magnetization. Figure 6 shows the theoretical curve corresponding to Eq. (58) for the repulsive Hubbard model at $M=0$ versus numerical results obtained by means of the DMRG technique for open chains of up to $L=128$ sites. The agreement between the analytical expression and numerical results is quite good, especially taking into account the fact that our analytical result (58) concerns only the $\sim L^{2}$ contribution.

Similar to the case of spin chains, one can study the tilt FS $\chi_{\mathcal{P}}$ (i.e., the response to the perturbation determined by

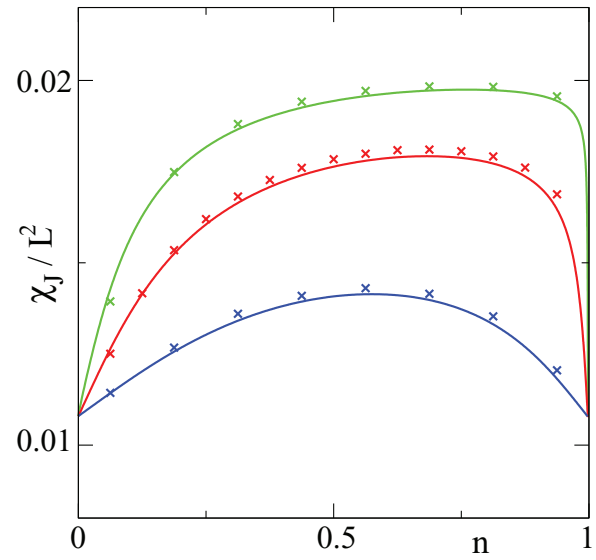

FIG. 6. (Color online) Current fidelity susceptibility of the fermionic Hubbard model with o.b.c. for $U / t=1,2$ and 6 (top to bottom). Symbols denote the DMRG data for Hubbard chains of $L=128$ sites. The lines correspond to the analytical expression (58).

$\left.\widehat{W}=\sum_{j} j n_{j}\right)$ of the fermionic Hubbard model with gapless charge excitations. Physically, such a perturbation can be either the lattice tilt (for atoms in optical lattices) or simply the external electric field (for charged particles). Proceeding in a close analogy to Sec. II E, we obtain

$$
\chi_{\mathcal{P}}=\frac{31 K_{c} L^{4} \zeta(5)}{4 u_{c}^{2} \pi^{6}} .
$$

Finally, a few remarks are in order concerning the behavior of Hubbard chains with p.b.c. One can again use the general connection between the current FS and the conductivity, as we have done for spin chains, but now the connection is between the charge current FS and charge conductivity, respectively. In the repulsive Hubbard model at half filling and at any magnetization, the charge excitations are gapped, so we expect the linear scaling of CFS independent of boundary conditions. Away from the half filling, using the low-frequency result for the conductivity of doped Mott insulators $\sigma_{\text {reg }}(\omega) \sim \omega^{3},{ }^{22}$ one again obtains a linear finite-size scaling, $\chi_{J}^{p} \sim L$, for any filling $v$ and magnetization $M$. We also expect the same behavior (linear scaling of the CFS with $L$ ) for the attractive case away from half filling. For the attractive two-component fermions at half filling (where umklapp is commensurate but irrelevant), however, using the perturbative result ${ }^{17} \sigma_{\text {reg }}(\omega) \sim U^{2} \omega^{4 K_{c}-5}$, one obtains

$$
\chi_{J}^{p} \sim L, \quad K_{c} \geqslant 5 / 4, \quad \chi_{J}^{p} \sim L^{6-4 K_{c}}, \quad 1<K_{c}<5 / 4 .
$$

The Hubbard model (with purely on-site attraction) is special since at half filling the charge sector enjoys $S U(2)$ symmetry, ${ }^{23}$ fixing $K_{c}=1$ (umklapp is marginally irrelevant), leading to the behavior described by Eq. (24). Hence one has to consider an extended Hubbard model for two-component attractive fermions to check Eq. (60).

\section{CURRENT FS IN GAPPED SYSTEMS}

Up to now, we have mostly dealt with systems that have a gapless excitation spectrum (except for the comment on the Hubbard model at half filling in the previous section). It is easy to see that the system size dependence of the CFS 
in gapped systems is generically linear, $\chi_{J} \propto L$, independent of boundary conditions. The reason is that the Drude part disappears in gapped phases, and the conductivity vanishes at energies below the excitation gap $\omega_{0}$ (since no excited states are available below the gap $), \sigma_{1}(\omega) \sim \Theta\left(\omega-\omega_{0}\right)$, where $\Theta(x)$ is the Heaviside step function. Alternatively, for systems with o.b.c., the unitary transformation approach of Sec. II D (which is applicable in the case of a pure chain geometry, i.e., in the absence of next-nearest-neighbor and longer-range hoppings) can be utilized for gapped spin chains as well and leads to formulas (40) and (41), which connect the CFS and the reduced longitudinal spin-spin correlator. In a gapped system (for example, in the Néel state of the spin- $\frac{1}{2} X X Z$ chain at $\Delta>1$ or in the Néel and rung-singlet phases of the spin- $\frac{1}{2}$ $X X Z$ ladder; see below), this correlator decays exponentially, so the sum in (41) will be proportional to $L$. A similar argument can be applied for fermionic or bosonic models.

Numerically, if the gap is extremely small, it may be difficult to distinguish exponential decay from algebraic decay; for the FS this would mean distinguishing the linear scaling $\chi_{J} \propto C L$ with a large prefactor $C$ from the quadratic scaling, $\chi_{J} \propto L^{2}$.

We illustrate the generic behavior of the CFS in gapped systems on the example of the spin- $\frac{1}{2}$ antiferromagnetic spin ladder defined by the Hamiltonian

$$
\begin{aligned}
\widehat{H}_{\mathrm{Lad}}= & \sum_{l, \alpha}\left[S_{l, \alpha}^{x} S_{l+1, \alpha}^{x}+S_{l, \alpha}^{y} S_{l+1, \alpha}^{y}+\Delta S_{l, \alpha}^{z} S_{l+1, \alpha}^{z}\right] \\
& +J_{R} \sum_{l} \vec{S}_{l, 1} \cdot \vec{S}_{l, 2}+d \sum_{l, \alpha}\left(\vec{S}_{l, \alpha} \times \vec{S}_{l+1, \alpha}\right)^{z},
\end{aligned}
$$

where $\alpha=1,2$ denotes the two legs of the ladder. In Fig. 7, we show the DMRG results for the CFS in the vicinity of the Ising quantum phase transition between the Néel and rung-singlet states. Ordinary quadratic scaling of the CFS peak at transition and linear scaling of the wings are observed.

However, there are peculiar cases when the CFS may have a nontrivial finite-size scaling in a gapped system with open

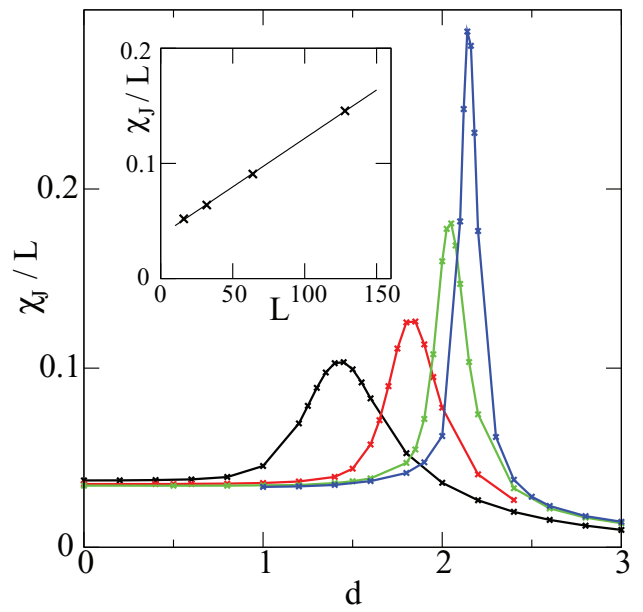

FIG. 7. (Color online) The CFS of a spin- $\frac{1}{2}$ antiferromagnetic ladder defined by (61), with o.b.c., in the vicinity of the Ising phase transition from the Néel to the rung-singlet state for $J_{R}=3$ and $\Delta=1.5$ and system sizes $L=16,32,64$, and 128 rungs. The inset shows that the peak of the CFS per site at the transition point scales linearly with the system size. boundaries. Namely, in a topologically ordered system, the presence of entangled edge spins localized at the boundaries may render the sum in $(41) \sim L^{2}$, despite the exponentially decaying correlation function. Let us take the spin-1 Haldane chain as an example. The topologically ordered ${ }^{24,25}$ ground state of the open Haldane chain is nearly fourfold degenerate ${ }^{26}$ due to the presence of spin- $\frac{1}{2}$ edge spins localized at the boundaries: the lowest state is a singlet, which is split from the Kennedy-Tasaki triplet by the exponentially small "boundary gap" $\propto e^{-L / \xi}$, where $\xi \sim 6$ is the bulk correlation length. In the singlet ground state, the reduced correlator between the edge spins remains finite, so, according to (41), $\chi_{J} \propto L^{2}$. It is clear that this behavior will be typical for any state characterized by the presence of edge spins that are nonlocally entangled with each other.

The scaling of $\chi_{J}$ will be very sensitive to the numerical errors; for example, if one accidentally takes a nonentangled member $|\uparrow \uparrow\rangle$ of the Kennedy-Tasaki triplet as the ground state, the reduced correlator between the edge spins will become zero, resulting in the generic linear behavior $\chi_{J} \propto L$.

\section{SUMMARY}

Combining numerical simulations with analytical arguments based on bosonization, we have studied the finite-size scaling of the current fidelity susceptibility $\chi_{J}$ with respect to the charge or spin currents in one-dimensional lattice models.

For both periodic and open boundary conditions DMRG scaling was performed for different matrix dimensions $m$ until a good convergence of fidelity susceptibility within four significant figures was reached (for larger system sizes up to $m \sim 1000$ was needed for periodic chains, although much less for open chains).

We related current fidelity susceptibility to the lowfrequency behavior of the corresponding conductivity and identified the main reason for different scaling laws in the gapless systems with open and periodic boundary conditions as the absence of the zero mode of the current operator for the former case. For systems with p.b.c. $\chi_{J}$ is directly connected to the low-frequency behavior of the regular part of the conductivity, while in open systems the leading system size dependence of $\chi_{J}$ is determined by the singular part of the conductivity that is essentially the smeared Drude peak.

For the systems with o.b.c. we obtained the universal quadratic scaling $\chi_{J} \propto L^{2}$, which obscures the detection of quantum phase transitions between two gapless regions by studying the finite-size scaling of the peak in $\chi_{J}$. Furthermore, for open chains we related $\chi_{J}$ with the tilt fidelity susceptibility that describes the response to the gradient of the chemical potential.

In future studies, it would be very helpful to perform numerical calculations of $\chi_{J}$ for large periodic spin- $\frac{1}{2} X X Z$ chains $L>100$ to confirm further the leading nontrivial low-frequency behavior of the regular part of the conductivity predicted by Giamarchi ${ }^{17}$ for $\Delta \lesssim 1$. It could also be interesting to study $1 \mathrm{D}$ models with infinite-size methods such as infinite, time evolving block decimation algorithm ${ }^{27}$ or infinite size $\mathrm{DMRG}^{28}$ and to determine the scaling of $\chi_{J}$ with the matrix dimension. Similar studies in higher dimensions could also be interesting. 


\section{ACKNOWLEDGMENTS}

We thank Eric Jeckelmann for helpful discussions. This work has been supported by QUEST (Center for Quantum Engineering and Space-Time Research) and DFG Research Training Group (Graduiertenkolleg) 1729.

\section{APPENDIX}

In this appendix, we derive analytical expressions for matrix elements of the total momentum $\int_{0}^{L} \tilde{\Pi} d x$ and the center-ofmass operator $\int_{0}^{L} x \partial_{x} \tilde{\Phi}(x) d x$ between the vacuum and excited states of the Gaussian bosonic model, with fields vanishing at the boundary $x=0$ and $x=L$.

We start with the total momentum operator. It is convenient to expand the bosonic fields in the Fourier modes of the open string,

$$
\begin{aligned}
& \tilde{\Phi}(x)=\sqrt{\frac{2}{L}} \sum_{n=1}^{\infty} \sin \frac{\pi n x}{L} \tilde{\phi}_{n}, \\
& \tilde{\Pi}(x)=\sqrt{\frac{2}{L}} \sum_{n=1}^{\infty} \sin \frac{\pi n x}{L} \tilde{\pi}_{n},
\end{aligned}
$$

which guarantees vanishing boundary conditions for fields $\tilde{\Phi}(0)=\tilde{\Pi}(0)=\tilde{\Phi}(L)=\tilde{\Pi}(L)=0$. The inverse relations,

$$
\begin{aligned}
& \tilde{\phi}_{n}=\sqrt{\frac{2}{L}} \int_{0}^{L} \sin \frac{\pi n x}{L} \tilde{\Phi}(x) d x, \\
& \tilde{\pi}_{n}=\sqrt{\frac{2}{L}} \int_{0}^{L} \sin \frac{\pi n x}{L} \tilde{\Pi}(x) d x,
\end{aligned}
$$

imply that zero modes for the open chain do not exist,

$$
\tilde{\phi}_{0} \equiv 0, \quad \tilde{\pi}_{0} \equiv 0 .
$$

Commutation relations of the Fourier modes are canonical,

$$
\left[\tilde{\phi}_{n}, \tilde{\pi}_{m}\right]=i \delta_{n, m} .
$$

The total momentum operator in terms of the Fourier components can be rewritten as

$$
\begin{aligned}
\int_{0}^{L} \tilde{\Pi} d x & =\int_{0}^{L} \sqrt{\frac{2}{L}} \sum_{n=1}^{\infty} \sin \frac{\pi n x}{L} \tilde{\pi}_{n} d x \\
& =\sqrt{2 L} \sum_{n=1}^{\infty} \frac{1-(-1)^{n}}{\pi n} \tilde{\pi}_{n},
\end{aligned}
$$

and the Luttinger liquid Hamiltonian reads

$$
\begin{aligned}
\widehat{H}_{L L} & =\frac{u}{2} \int_{0}^{L}\left[\left(\partial_{x} \tilde{\phi}\right)^{2}+\tilde{\Pi}^{2}\right] d x=\frac{u}{2} \sum_{n=1}^{\infty}\left[\tilde{\pi}_{n}^{2}+\left(\frac{\pi n}{L}\right)^{2} \tilde{\phi}_{n}^{2}\right] \\
& =\frac{\pi u}{L} \sum_{n>0} n\left[\tilde{a}_{n}^{\dagger} \tilde{a}_{n}+\frac{1}{2}\right]=\sum_{n>0} \omega_{n} \tilde{a}_{n}^{\dagger} \tilde{a}_{n}+E_{0} .
\end{aligned}
$$

Here

$$
\tilde{a}_{n}^{\dagger}=\sqrt{\frac{L}{2 \pi n}} \tilde{\pi}_{n}+i \sqrt{\frac{\pi n}{2 L}} \tilde{\phi}_{n}, \quad \tilde{a}_{n}=\sqrt{\frac{L}{2 \pi n}} \tilde{\pi}_{n}-i \sqrt{\frac{\pi n}{2 L}} \tilde{\phi}_{n}
$$

are the standard bosonic creation and annihilation operators, $\tilde{a}_{n}|0\rangle=0$, satisfying the commutation relations $\left[\tilde{a}_{n}, \tilde{a}_{m}^{\dagger}\right]=$ $\delta_{n, m}$ and defining the eigenstates $\widehat{H}_{L L} \tilde{a}_{n}^{\dagger}|0\rangle=\left(\omega_{n}+E_{0}\right) \tilde{a}_{n}^{\dagger}|0\rangle$.

The total momentum operator in this basis obtains the following form:

$$
\int_{0}^{L} \tilde{\Pi} d x=\sum_{n} \frac{1-(-1)^{n}}{\sqrt{\pi n}}\left[\tilde{a}_{n}+\tilde{a}_{n}^{\dagger}\right] .
$$

Hence,

$$
\left\langle n\left|\int_{0}^{L} \tilde{\Pi} d x\right| 0\right\rangle=\frac{1-(-1)^{n}}{\sqrt{\pi n}} .
$$

The matrix elements of the fermionic total current are obtained from the relation

$$
\widehat{J} \rightarrow \frac{\sqrt{K} u}{\sqrt{\pi}} \int_{0}^{L} \tilde{\Pi} d x,
$$

so that

$$
\begin{aligned}
\rho(n)\left|\left\langle\psi_{n}|\widehat{J}| \psi_{0}\right\rangle\right|^{2} & =\frac{K u^{2}}{\pi}\left|\left\langle n\left|\int_{0}^{L} \tilde{\Pi} d x\right| 0\right\rangle\right|^{2} \\
& =\frac{K u^{2}\left[1-(-1)^{n}\right]^{2}}{\pi^{2} n} .
\end{aligned}
$$

Note that in the bosonized formulation for each excitation $E_{n}=u \pi n / L+E_{0}$ there is a corresponding single state $|n\rangle=$ $\tilde{a}_{n}^{\dagger}|0\rangle$ obtained from the vacuum by acting with the total momentum operator; in other words, the bosonic density of states is $\rho_{\text {bosonic }}(n)=1$, as opposed to the fermionic picture where $\rho(n)=n$.

In a similar way, we can calculate the matrix elements of the polarization (center-of-mass) operator

$$
\widehat{\mathcal{P}}=\sum_{j} j S_{j}^{z} \mapsto-\sqrt{\frac{K}{\pi}} \int_{0}^{L} x \partial_{x} \tilde{\Phi}(x) d x .
$$

One obtains

$$
\begin{aligned}
-\int_{0}^{L} x \partial_{x} \tilde{\Phi}(x) d x & =-\sum_{n} \frac{\sqrt{2 L}}{\pi n} \tilde{\phi}_{n} \int_{0}^{\pi n} y \cos y d y \\
& =\sqrt{2 L} \sum_{n} \frac{1-(-1)^{n}}{\pi n} \tilde{\phi}_{n} .
\end{aligned}
$$

On the other hand,

$$
\tilde{\phi}_{n}=\sqrt{\frac{2 L}{\pi n}} \frac{\tilde{a}_{n}^{\dagger}-\tilde{a}_{n}}{2 i} ;
$$

hence,

$$
-\int_{0}^{L} x \partial_{x} \tilde{\Phi}(x) d x=L \sum_{n} \frac{1-(-1)^{n}}{(\pi n)^{3 / 2}} \frac{\tilde{a}_{n}^{\dagger}-\tilde{a}_{n}}{i} .
$$

Using similar reasoning as for deriving Eq. (A9), we arrive at the generalization of Eq. (52) for the interacting case:

$$
\rho(n)\left|\left\langle\psi_{n}|\widehat{\mathcal{P}}| \psi_{0}\right\rangle\right|^{2}=\frac{K L^{2}\left[\left(1-(-1)^{n}\right)\right]^{2}}{\pi^{4} n^{3}} .
$$


${ }^{1}$ W.-L. You, Y.-W. Li, and S.-J. Gu, Phys. Rev. E 76, 022101 (2007).

${ }^{2}$ L. Campos Venuti and P. Zanardi, Phys. Rev. Lett. 99, 095701 (2007).

${ }^{3}$ D. Schwandt, F. Alet, and S. Capponi, Phys. Rev. Lett. 103, 170501 (2009).

${ }^{4}$ S.-J. Gu, Int. J. Mod. Phys. B 24, 4371 (2010).

${ }^{5}$ P. Zanardi and N. Paunkovic, Phys. Rev. E 74, 031123 (2006).

${ }^{6}$ S. R. White, Phys. Rev. Lett. 69, 2863 (1992).

${ }^{7}$ U. Schollwöck, Rev. Mod. Phys. 77, 259 (2005).

${ }^{8}$ M. Thesberg and E. S. Sørensen, Phys. Rev. B 84, 224435 (2011)

${ }^{9}$ B. S. Shastry and B. Sutherland, Phys. Rev. Lett. 65, 243 (1990).

${ }^{10}$ A. A. Zvyagin, Fiz. Tverd. Tela (Leningrad) 32, 1546 (1990) [Sov. Phys. Solid State 32, 905 (1990)].

${ }^{11}$ T. Giamarchi, Quantum Physics in One Dimension (Oxford University Press, Oxford, 2003).

${ }^{12}$ D. Pines and P. Nozieres, Theory of Quantum Liquids (Benjamin, New York, 1966).

${ }^{13}$ F. H. L. Essler, F. Gebhard, and E. Jeckelmann, Phys. Rev. B 64, 125119 (2001).
${ }^{14}$ E. Jeckelmann, Phys. Rev. B 66, 045114 (2002).

${ }^{15}$ C. N. Yang and C. P. Yang, Phys. Rev. 150, 321 (1966).

${ }^{16}$ B. Hetényi, Phys. Rev. B 87, 235123 (2013).

${ }^{17}$ T. Giamarchi, Phys. Rev. B 44, 2905 (1991).

${ }^{18}$ S. Greschner, L. Santos, and T. Vekua, Phys. Rev. A 87, 033609 (2013).

${ }^{19}$ M. F. Yang, Phys. Rev. B 76, 180403(R) (2007).

${ }^{20}$ T. Hikihara and A. Furusaki, Phys. Rev. B 69, 064427 (2004).

${ }^{21}$ H. J. Mikeska and W. Pesch, Z. Phys. B 26, 351 (1977).

${ }^{22}$ T. Giamarchi and A. J. Millis, Phys. Rev. B 46, 9325 (1992).

${ }^{23}$ F. H. L. Essler, H. Frahm, F. Göhmann, A. Klümper, and V. Korepin, The One-Dimensional Hubbard Model (Cambridge University Press, Cambridge, 2003).

${ }^{24}$ M. den Nijs and K. Rommelse, Phys. Rev. B 40, 4709 (1989).

${ }^{25}$ S. M. Girvin and D. P. Arovas, Phys. Scr. T 27, 156 (1989).

${ }^{26}$ T. Kennedy and H. Tasaki, Phys. Rev. B 45, 304 (1992); Commun. Math. Phys. 147, 431 (1992).

${ }^{27}$ G. Vidal, Phys. Rev. Lett. 98, 070201 (2007).

${ }^{28}$ I. P. McCulloch, arXiv:0804.2509. 